\title{
4D Printing of Smart Stimuli-Responsive Polymers
}

\author{
Raffaella Suriano, $\oplus^{\mathrm{z}}$ Roberto Bernasconi, ${ }^{*}$ Luca Magagnin, ${ }^{* * *}$ and Marinella Levi $₫$ \\ Department of Chemistry, Materials and Chemical Engineering “Giulio Natta,” Politecnico di Milano, Milan, Italy
}

\begin{abstract}
This paper starts with a short review of smart materials, which can be 4D printed and whose properties can be modified over time through specific stimuli such as heat, light, temperature, electric and magnetic fields. In the second part of the paper, a new polymer material for $4 \mathrm{D}$ printing was presented. The material was a thermally activated shape memory polymer (SPM), capable of memorizing a temporary shape and restoring the original one by means of a thermal stimulus. A self-healing behavior was introduced into the polymer, to enhance its durability and make it suitable for application in the fields of soft robotics and actuators. A polycaprolactone (PCL) was selected to provide a shape memory effect, taking into account its molecular weight and the possibility of chemically modifying it in order to make it photo-curable. Self-healing properties were provided by an ureidopyrimidinone (UPy) methacrylate monomer, capable of forming four hydrogen bonds with a self-complementary unit, favored by temperature. This new material was thus 4D printed by a digital liquid processing (DLP) printer. Both the shape memory effect and the self-healing feature were demonstrated, enabling this new material to be used as a raw material for new generation soft robotic actuators.

(C) The Author(s) 2019. Published by ECS. This is an open access article distributed under the terms of the Creative Commons Attribution Non-Commercial No Derivatives 4.0 License (CC BY-NC-ND, http://creativecommons.org/licenses/by-nc-nd/4.0/), which permits non-commercial reuse, distribution, and reproduction in any medium, provided the original work is not changed in any way and is properly cited. For permission for commercial reuse, please email: oa @ electrochem.org. [DOI: 10.1149/2.0411909jes]

(cc) BY-NC-ND
\end{abstract}

Manuscript submitted March 11, 2019; revised manuscript received May 14, 2019. Published June 3, 2019. This paper is part of the JES Focus Issue on 4 D Materials and Systems.

The 4D printing concept was recently introduced to define the 3D printing of objects that are capable of changing their shape over time due to either a smart involvement of multiple materials in a single print or additive manufacturing of smart stimuli-responsive materials. ${ }^{1-3}$ The incorporation of the fourth dimension into common 3D printing process is essential when printed components are able to switch between multiple configurations triggered by external stimuli, e.g. temperature, light, pressure, moisture, magnetic or electric field. ${ }^{4}$ Accordingly, the fourth dimension is time needed by the smart materials to actuate their properties, to reshape themselves or self-assemble. ${ }^{5}$ One of the most diffused 4D printable stimuli-sensitive materials are shape memory materials, particularly shape memory polymers (SMPs). ${ }^{6,7}$

SMPs are a class of intelligent polymers able to change their shape by means of a stimulus. Indeed, SMPs can be deformed and fixed into a temporary shape that can be turned into the permanent one by applying a stimulus. ${ }^{8}$ Compared to the well-known shape memory alloys (SMAs), SMPs present some important advantages, such as a high strain recovery, low density, cheapness, potential chemical stability, easier processability, potential recyclability, biocompatibility, and biodegradability. ${ }^{9}$ Moreover, the recovery of the original shape is faster than SMAs, due to the higher permeability to air. ${ }^{10}$ These polymeric systems consist of two segments: the netpoints and a switching phase. The switching segments can be crystallites or glassy domains, while the netpoints can be based on either chemical (i.e. covalent bonds) or physical (i.e. intermolecular interactions) crosslinks, determining the classification in physically or chemically crosslinked SMPs. A prerequisite of the system is sufficient elastic deformability, in order to have the flexibility of the switching segments during the recovery of the permanent shape. Moreover, a minimum amount of netpoints is required to fix the temporary shape in the imposed position, simultaneously with the solidification of the switching segments. A programming process defines the fixing of a temporary shape. In case of a thermally induced shape memory effect (SME), the material is heated up to a $\mathrm{T}>\mathrm{T}_{\text {trans }}$, where the $\mathrm{T}_{\text {trans }}$ is the temperature at which the switching segments melt or become viscous. The nature of the $\mathrm{T}_{\text {trans }}$ is determined by the type of the switching segments:

\footnotetext{
*Electrochemical Society Student Member.

**Electrochemical Society Member.

${ }^{\mathrm{z}}$ E-mail: raffaella.suriano@polimi.it
}

glassy domains will become viscous at $\mathrm{T}>\mathrm{T}_{\mathrm{g}}\left(\mathrm{T}_{\text {trans }}=\mathrm{T}_{\mathrm{g}}\right)$; crystallites can be melted at $\mathrm{T}>\mathrm{T}_{\mathrm{m}}\left(\mathrm{T}_{\text {trans }}=\mathrm{T}_{\mathrm{m}}\right)$. In this state, the material can be transformed into a temporary shape, which can be fixed by cooling down to a $\mathrm{T}<\mathrm{T}_{\text {trans }}$, due to the presence of netpoints and to the crystallization/vitrification of the switching segments. By reheating, either crystallites or glassy domains respectively melt or return to the viscous state, leading to the regain of the original permanent shape. ${ }^{8,10,11}$

Extrusion-based printing of shape memory and stimuliresponsive polymers.-Among several printing technologies explored so far, fused deposition modeling (FDM)-based printing is the most popular and widespread method, which creates objects layer-by-layer by extruding molten thermoplastic polymers as well as ceramics and metal pastes. ${ }^{12}$ FDM technology was also employed to fabricate 4D printed structures, made of polylactic acid (PLA), which showed an SME due to the change of chain mobility at the glass transition temperature. ${ }^{13}$ Indeed, amorphous domains of PLA are switching domains, while the crystalline domains act as permanent netpoints stabilizing the original shape. ${ }^{14}$ Yang et al. reported the use of an FDM process to print structures with shape memory properties, made of a thermoplastic polyurethane elastomer, named DiAPLEX MM-4520. ${ }^{15}$ $4 \mathrm{D}$ printable filaments of this material were obtained by extruding pellets provided by SMP Technologies, Inc.

Another extrusion-based technology, like FDM, is direct ink writing (DIW), ${ }^{16}$ also called liquid deposition modeling (LDM). ${ }^{17}$ In order to build and produce 3D structures, rheological properties of inks or liquids extruded by a syringe and deposited in layers play a crucial role. Dispersion, colloidal suspensions and other mixtures are usually employed to produce viscoelastic inks and print objects composed of ceramic materials, polymers, and nanocomposites. Thermoresponsive hydrogels were also printed by a UV-assisted LDM system to fabricate $4 \mathrm{D}$ soft structures, which can be used as thermally switching sensors and actuators. ${ }^{18}$ In another work performed by Gladman et al., localized anisotropic swelling was exploited to induce changes in hydrogel complex shapes 4D printed by DIW on immersion in water. ${ }^{19}$ A successful approach for the 4D printing of shape memory polymers was the extrusion of UV cross-linkable PLAbased ink by a UV-assisted DIW to produce shape-changing SMP architectures and magnetically triggered nanocomposite for biomedical applications. $^{20}$ 
Inkjet printing and $4 \mathrm{D}$ printing of shape memory materials.Another promising technique to produce 4D materials is inkjet printing. This technology allows the deposition and patterning of materials in the form of solutions or particles suspensions by emitting micrometric droplets at high speed from a nozzle. ${ }^{21,22}$ Droplets travel from the nozzle to a substrate, where they are deposited in lines and then in patterns. Moreover, by printing more layers one on top of the others, the thickness of the deposited layers can be controlled efficiently. The possibility of printing low viscosity solutions allows the deposition of a wide range of materials, including polymers, ${ }^{23,24}$ sol-gels, ${ }^{25,26}$ and dyes. ${ }^{27}$ Conversely, inkjet ability to print particles suspensions makes possible also the deposition of insoluble materials like metals, ${ }^{28}$ metal oxides ${ }^{29,30}$ and ceramics ${ }^{31}$ in the form of nanoparticles. Moreover, some organic functional materials like graphene ${ }^{32}$ or carbon nanotubes ${ }^{33}$ can be easily printed, and even living cells. ${ }^{34}$ Applications of materials inkjet printing include flexible electronics, ${ }^{35}$ sensors, ${ }^{36,37}$ microfluidic setups, ${ }^{38}$ optoelectronic devices ${ }^{39}$ and many others. Its high precision and low cost open interesting sceneries also in the field of microfabrication. ${ }^{22}$

Despite the possibility of controlling layer thickness, inkjet printing is normally intrinsically bidimensional. It is, in fact, ideal to obtain thin layers characterized by great uniformity on even large surfaces while developing geometries in the third dimension is more critical. This fact is related to the fluidic nature of the droplets generated by the nozzle. Recently, however, 2.5D and 3D inkjet methodologies have been developed. A first simple approach is the superimposition of a large number of printing layers, which is possible with some materials. Inkjet tridimensional printing was demonstrated with metal nanoparticles following this method. ${ }^{40,41}$ Moreover, living cells have been printed superimposing many layers. ${ }^{42}$ Manufacturing possibilities are however strongly limited in the third dimension, giving origin to structures developing mainly in $2.5 \mathrm{D}$. In some cases, printing of highly concentrated solutions is possible, resulting in truly $3 \mathrm{D}$ parts. This is the case of ceramic nanoparticles containing inks. ${ }^{43}$ Besides standard inkjet, real 3D inkjet printing techniques are available as well. The most important is Polyjet, ${ }^{44}$ which is based on the in-situ crosslinking (using UV light) of a photopolymer emitted in the form of droplets from a nozzle. Tridimensionality is greatly enhanced and complex parts can be easily manufactured. Moreover, multi-material printing can be achieved using multiple nozzles for different materials.

Some of these innovative 2.5D and 3D inkjet techniques has been extended also to include the time domain, yielding the first significant examples of inkjet printed SMPs and materials in general. From this point of view, the most interesting technique is Polyjet multi-material inkjet printing. This technology can be efficiently applied to 4D materials fabrication, as Ding et al. recently demonstrated. ${ }^{45}$ They manufactured composites shapes with programmed time evolution upon heating. One of the most important examples of SMPs fabrication via Polyjet is the one reported by Raviv et al., who produced self-evolving structures able to deform in response to an external stimulus. ${ }^{46} \mathrm{Wu}$ et al. printed active shape memory polymeric composites, ${ }^{47}$ while Yap et al. printed SMPs in the form of honeycomb structures. ${ }^{48}$

Other notable examples include the work by Wagner et al., which used Polyjet printing to build shape-transforming auxetic structures. ${ }^{49}$ $\mathrm{Ge}$ et al. used the same technique to make active origami. ${ }^{3}$ Polyjet can be also used to embed functional shape memory components to impart actuation functions to $3 \mathrm{D}$ printed parts, as demonstrated by Meisel et al. ${ }^{50}$

Standard inkjet printing can be used as well for SMPs manufacturing, as demonstrated by Van Oosten et al., who printed light-driven artificial cilia from liquid-crystal containing solutions. ${ }^{51}$ Normal inkjet, however, is more notably employed for the realization of other kinds of 4D materials. Different authors, for example, employed inkjet printing for the realization of RF electronics, including antennas ${ }^{52}$ or energy harvesting sensors. ${ }^{53}$ Standard inkjet can be also used to impart 4D functionalities to 2D or 3D structures, such as Miura structures built by the group of M.M. Tentzeris. Such structures were produced starting from a $2 \mathrm{D}$ paper origami-like structure, which acquires the ability to change shape thanks to dipole elements printed on the folding lines of the paper structure ${ }^{54,55}$ Peng et al. followed a similar approach to apply 4D functionalities to 3D hydrogel structures. ${ }^{56}$ Moreover, standard inkjet can be used as a complementary technique for other 4D printing techniques. Zarek et al., for example, stereolithography-printed shapechanging structures and subsequently inkjet-printed conductive tracks to realize a $4 \mathrm{D}$ electrical circuit. ${ }^{57}$ Inkjet $4 \mathrm{D}$ printing of biomaterials is another interesting research topic, with some fascinating examples. ${ }^{58}$ Villar et al. also printed living cells network programmable by osmosis gradients to fold in controlled ways. ${ }^{59}$

Digital liquid processing and stereolithography of $4 D$ printable materials. - In addition to inkjet printing, other photopolymerizationbased methods have been widely used for the 4D printing of SMPs, such as digital liquid processing (DLP) and stereolithography (ST). The first work about the 3D printing of a methacrylated polycaprolactone (PCL) with SME by a DLP printer was presented by Zarek et al., who demonstrated the capability of this PCL-based materials to be integrated into a flexible and stimuli-responsive electrical device. ${ }^{57}$ This preliminary study paved the way for other potential applications of 4D printed materials, such as for customized medical devices ${ }^{60}$ responsive jewelry, and fashion accessories. ${ }^{61}$ However, a limitation of PCL-based materials can be biodegradability and mechanical degradation over repeated thermomechanical cycles. Choong et al. reported the stereolithography of an SMP composed of crosslinked acrylates with good durability of SMP properties and prolonged thermomechanical cycle life. ${ }^{62}$

SMPs, thanks to their elasticity and deformability, can be employed as flexible actuators, to replicate the functionality of muscles in animals. In fact, a muscle-like structure has a larger number of degrees of freedom compared to hard robotics, with their deformability and compliance, making them suitable for the human-machine interactions. ${ }^{63,64}$ Therefore, the aim of the so-called soft robotics is to incorporate soft bioinspired technologies into robots to permit them to adapt to unpredictable environments in the presence of humans. ${ }^{65}$ For example, soft materials could reduce the harm inadvertently caused by robotic systems, or improve the grasping. In particular, smart materials like SMPs embed the need for softness and body compliance with the actuation capability, making the SME one of the most used actuation technique in soft robotics. ${ }^{65}$

In the light of scientific studies mentioned above, a novel smart material was developed in this work, using an SMP with the ability to self-repair due to a specific self-healing provider. This ability is a benefit for a potential application in soft robotics because it enhances the material durability by mimicking natural tissues. A PCL dimethacrylate was selected as a photocurable SMP and selfhealing properties were provided by a methacrylate monomer with a 2-ureido- $4[1 \mathrm{H}]$-pyrimidinone functionality, due to its ability to form stable supramolecular interactions favored by heat. ${ }^{66} 4 \mathrm{D}$ printing of this material was successfully achieved, opening the possibility to create customized complex geometries. The printing process was performed by modifying a low-cost DLP printer. The potential application of this newly prepared material for the soft robotic actuation was demonstrated by printing and damaging an opposite thumb. SME was still preserved after the healing process, confirming the feasibility of using this material for human-machine interactions.

\section{Experimental}

Components of the blend.-The materials discussed from here on are polycaprolactone with a molecular weight of $10000 \mathrm{~g} / \mathrm{mol}$ (PCL10K) and UPy methacrylate monomer, from here on referred to as UPyMA.

End-capping reaction of PCL.-PCL diol was end-capped with 2-isocyanatoethyl methacrylate (2-IEM) in order to make it photocurable and $3 \mathrm{D}$ printable. Considering that 2 -IEM is very reactive in presence of $\mathrm{OH}$ groups, PCL10K was dried for 2 hours at $120^{\circ} \mathrm{C}$ under dynamic vacuum in order to remove any traces of humidity in the polymer and in the round-bottom flask. Then, the system was 
cooled down to $60^{\circ} \mathrm{C}$. At this point, $1 \mathrm{~mL}$ of chloroform for each $1.2 \mathrm{~g}$ of PCL10K was added in order to decrease the viscosity and favor the mixing between the reagents.

Once 2-IEM was added to the round-bottom flask, a 2\%wt. of tin octanoate (Sigma-Aldrich) was also added with respect to the amount of PCL10K and used as a catalyst. Both reagents were dissolved in a minimum quantity of chloroform. Usually, the ratio between 2-IEM and PCL varies between 2.2 and 3, as reported in the work of Messori et al., ${ }^{67}$ but in this case, a ratio of 1.9 was used in order to reduce as much as possible the unreacted quantity of 2-IEM. The reaction between PCL diol and IEM was monitored through FT-IR spectroscopy: every hour a drop was withdrawn from the round-bottom flask and put between two $\mathrm{NaCl}$ round disks. After two hours, the absorption peak at $2270 \mathrm{~cm}^{-1}$ associated with $\mathrm{N}=\mathrm{C}=\mathrm{O}$ functional group disappeared. The reaction was stopped and methacrylated PCL (PCLDMA) was precipitated in petroleum ether. This operation was carried out in order to eliminate completely any residual trace of 2-IEM. PCLDMA was separated from petroleum ether and then dried under a fumehood.

Methacrylation of UPy monomer.-The synthesis process to obtain a methacrylate monomer (UPyMA) designed by Yamauchi et al. was followed, ${ }^{68}$ with some modifications. UPy motifs, responsible for self-healing behavior due to their capability to establish selfcomplementary quadruple hydrogen bonding interactions, were incorporated into the polymer matrix through methacrylation reaction: a methacrylate group was added to UPy in order to allow its crosslinking with PCLDMA chains upon UV-vis light exposure. The solvent used was dimethyl sulfoxide (DMSO, Sigma-Aldrich), which was dehydrated by means of molecular sieves for 3 days under a nitrogen atmosphere. A white solid powder of methyl isocytosine (MIS) equal to the $4 \%$ wt in DMSO was added to a round bottom flask, which was previously sealed with a rubber septum and a condenser. $50 \mathrm{~mL}$ ( $55 \mathrm{~g}$ ) of DMSO were added to the system with a syringe. The temperature was increased to $170^{\circ} \mathrm{C}$ and the temperature inside the flask was constantly monitored by means of a thermometer. Once the desired temperature was reached in the flask, the dissolution of MIS solid powder was achieved. Afterward, the oil bath was rapidly removed and 2-IEM, equal to the 5\%wt. in DMSO, was added to the batch through a syringe, keeping the system under magnetic stirring. Then, the mixture needed to be cooled using an ice bath. This induced the precipitation of the UPyMA solid. The final methacrylated monomer was precipitated washing the solution in a large excess of acetone to remove any trace of unreacted 2-IEM monomer. Its composition was analyzed by ${ }^{1} \mathrm{H}-\mathrm{NMR}$ and peak integrations of the spectrum showed good agreement with the structure reported in the literature. ${ }^{68} \mathrm{DSC}$ analysis showed a melting temperature of $207^{\circ} \mathrm{C}$ for the synthesized UPyMA.

Photopolymerization reaction.-Once the two procedures previously described were carried out to obtain photopolymerizable components, the blend was prepared by dissolving PCLDMA and UPyMA in chloroform, $60 \%$ wt. with respect to the reactive parts of the system. UPyMA was added to a vial with chloroform and left stirred for $1 \mathrm{~h}$ in an oil bath heated up to $45^{\circ} \mathrm{C}$ to achieve its complete dissolution. Afterward, PCLDMA was transferred gradually into the same vial, always kept at $45^{\circ} \mathrm{C}$ and under stirring until the total disappearance of any trace of solid in suspension occurred. 2,4,6-trimethylbenzoylphenyl phosphinate, (TPO-L, IGM resins), was added to the system (4\% wt. with respect to the reactive parts of the system) and stirred magnetically for $5 \mathrm{~min}$ at room temperature. Then, the liquid precursor was cast into molds that were put under UV-A light to trigger photopolymerization. After $10 \mathrm{~min}$ of exposure ( $5 \mathrm{~min}$ for each side), a polymer network was finally achieved. The same procedure but without UPy methacrylated monomer has been performed: PCLDMA was melted at $60^{\circ} \mathrm{C}$ and after adding TPO-L the resulting solution was used to cast specimens of PCLDMA only for the same exposure time used for PCLDMA+UPy specimens.
Printing tests.-To 4D print the mixture of PCLDMA and UPyMA, a DLP printer (TOP DLP, Wasp Italy) was used with a topdown configuration. Compared to typical bottom-up configurations of SL printers, the projector of this DLP printer was located at the top of the printer, whereas the reservoir with the liquid photopolymerizable resin was under the projector. This printer was designed to work only at room temperature. In order to print the PCLDMA + UPyMA formulation at $45^{\circ} \mathrm{C}$, a custom-made hot-plate was developed and introduced in the printer during the entire printing process to keep the mixture at this temperature, which was necessary to obtain the best UPyMA diffusion into the material.

Chemical and thermal characterization.- ${ }^{1} \mathrm{H}$ NMR spectroscopy was exploited to determine the methacrylation degree (DM) of PCLDMA using a Bruker $400 \mathrm{MHz}$ spectrometer and $\mathrm{CDCl}_{3}$ as solvent. In particular, DM was determined according to the procedure described by Zarek and coworkers. ${ }^{57}$ The instrument used in this work for FTIR spectroscopy in absorption mode, at room temperature and in air was an FT/IR-615 (Jasco Inc.). DSC analyses were carried out with a DSC $823 \mathrm{e}$ (Mettler Toledo), heating the samples from $-50^{\circ} \mathrm{C}$ to $250^{\circ} \mathrm{C}$ with a heating rate of $10^{\circ} \mathrm{C} / \mathrm{min}$, then cooling them with a rate of $5^{\circ} \mathrm{C} / \mathrm{min}$ and heating again with a rate of $10^{\circ} \mathrm{C} / \mathrm{min}$.

Self-healing and tensile tests.-Stereo Microscope SZ40-CTV (Olympus) was used to evaluate surface self-healing abilities of materials by performing scratches with a scalpel blade on sample surfaces. The images were obtained with a $5 x$ objective and a camera controlled by means of Infinity Analyse 2 software (Lumenera Corp.).

Bulk self-repairing abilities were assessed by cutting half of the cross-sections and then putting samples in an oven at $80^{\circ} \mathrm{C}$ for $1 \mathrm{~h}$ to trigger the formation of self-complementary quadruple hydrogen bonding interactions between two UPy motifs. The two faces of the cut cross-sections were kept in close contact by applying pressure, in order to avoid the possible detachment of the faces during thermal treatment.

Mechanical properties of the virgin and healed materials such as Young's modulus and tensile strength were determined through uniaxial tensile tests. The dynamometer used to carry out the analyses was a Zwick/Roell Z010 (Zwick Roell, Germany) with a $10 \mathrm{kN}$ load cell and a longstroke extensometer. Tests were performed at two different displacement speeds, $3 \mathrm{~mm} \cdot \mathrm{min}^{-1}$ and $30 \mathrm{~mm} \cdot \mathrm{min}^{-1}$, and with a preload of $1 \mathrm{~N}$ at the same speed of the test.

\section{Results and Discussion}

Characterization of PCLDMA.-PCLDMA was analyzed through DSC in order to understand how the end-capping reaction influenced both the degree of crystallinity and the melting temperature of neat PCL. The degree of crystallinity $\chi$ was calculated as follows:

$$
\chi=\frac{\Delta H_{\text {endothermic DSC peak }}}{\Delta H_{100 \% \text { crystalline PCL }}}
$$

taking into account the endothermic peak of the second heating DSC scan and a melting enthalpy of $134.9 \mathrm{~J} / \mathrm{g}$ for a $100 \%$ crystalline PCL. It was found that end-capping of PCL with 2-IEM determined a reduction in the crystallinity degree of the polymer network from $47 \%$ to $37 \%$ and consequently in its melting temperature, which decreased from $57^{\circ} \mathrm{C}$ to $54^{\circ} \mathrm{C}$, as also shown in Figure 1 . This result was expected because the methacrylate end groups introduced small defects and irregularities in PCLDMA chains, leading to a reduced degree of crystallinity and a decrease of crystallite dimensions, with a consequent reduction of the melting temperature. Similar results were also reported in previous work, carried out by Lendlein and coworkers, ${ }^{69}$ who observed that the melting temperature for a PCL decreased from about $55^{\circ} \mathrm{C}$ to $50^{\circ} \mathrm{C}$ by introducing methacrylate end groups.

The degree of methacrylation achieved with this reaction was determined by ${ }^{1} \mathrm{H}-\mathrm{NMR}$ spectroscopy and it was equal to $74 \%$, leading to a gel content value of $96 \%$ in the crosslinked PCLDMA material. 


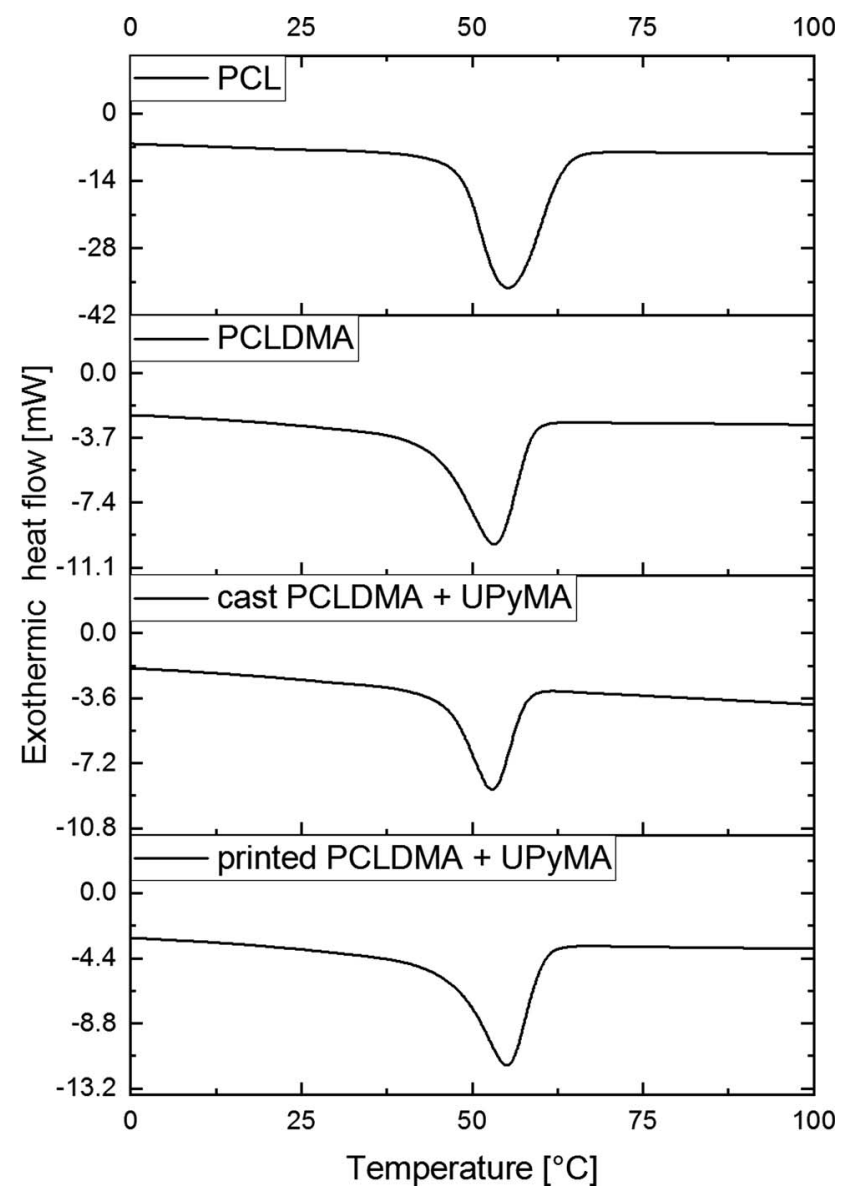

Figure 1. DSC heating thermograms obtained with a heating temperature of $10^{\circ} \mathrm{C} / \mathrm{min}$ for a neat $\mathrm{PCL}$, for a PCL end-capped with methacrylate groups (PCLDMA), for a cast sample composed of PCLDMA and UPyMA (4\% wt. with respect to PCLDMA weight) crosslinked by UV exposure and for a printed PCLDMA+UPyMA sample with the same composition of cast sample.

Effect of UPyMA concentration on self-healing properties.To obtain the best performances in terms of SME and self-repairing ability, some concentrations of UPyMA in the range of 3-5\% wt. with respect to the weight percentage of PCLDMA were considered. PCLDMA-UPyMA samples were cast in a bar mold and photocured. As regards the self-repairing assessment, the bars were damaged on the surface with a razor blade to produce a deep scratch and then were thermally treated for $1 \mathrm{~h}$ at $80^{\circ} \mathrm{C}$. Figure $2 \mathrm{a}$ shows the scratched surface of a rectangular specimen of the cast and photo-cured neat PCLDMA. After $1 \mathrm{~h}$ in the oven at $80^{\circ} \mathrm{C}$, the damage was still present (Figure $2 \mathrm{~b}$ ). After the introduction of UPy motifs, all the specimens showed selfhealing behavior. Independently from the amount of UPyMA, all the scratches disappeared after thermal treatment in an oven at $80^{\circ} \mathrm{C}$ for $1 \mathrm{~h}$ (Figures 2c-2h).

Analysis of shape memory effect.-The same range of UPyMA concentrations was considered to investigate shape memory properties. To verify the SME, the samples were programmed in a temporary stretched shape and then subjected to the recovery of the original shape. Increasing the temperature above the $T_{m}$ of PCLDMA, the specimens became flexible and semitransparent, due to the melting of the crystalline phases of PCLDMA that acted as switching domains. At temperatures higher than $\mathrm{T}_{\mathrm{m}}$, for example $80^{\circ} \mathrm{C}$, the sample was deformed into a desired temporary shape and kept in this configuration for some seconds. Then, the structure was cooled down and crystallization of PCLDMA crystalline domains fixed the temporary configuration also with the help of the hard domains, the crosslinked amorphous parts. The switching to the permanent shape occurred re- heating the sample at $60^{\circ} \mathrm{C}$. These qualitative tests were performed according to the protocol described by Behl and Lendlein. ${ }^{70}$ By increasing the amount of UPyMA monomer, the rectangular specimen required a higher amount of time to shift from the temporary to the permanent shape. A partial recovery of the permanent shape was observed for the sample with a 5\% wt of UPyMA that showed a deformed shape even after a long thermal treatment at $60^{\circ} \mathrm{C}$. Taking into account the results of the previous tests performed to evaluate the effect of UPyMA concentration on self-healing behavior and SME, 4\% wt. of UPyMA was selected as a final concentration. Indeed, this concentration assured shape memory properties but also allowed the system to benefit from a higher amount of UPy motifs, improving self-healing properties. Once found the optimal UPyMA percentage, DSC analysis was also performed for PCLDMA + UPyMA crosslinked sample to investigate how the presence of UPy influenced the melting temperature and the degree of crystallinity. DSC scans showed that the sample with UPyMA exhibited a melting temperature of around $53^{\circ} \mathrm{C}$ (Figure 1), and its degree of crystallinity decreased to $25 \pm 5 \%$, when compared to PCLDMA sample without UPyMA. This result can be explained, considering the presence of UPyMA, which introduced local irregularities in the material, thus hindering the crystallization of PCLDMA chains.

Self-healing and shape memory properties of $4 D$ printed structures.-The novel 4D printable self-healable and shape memory polymer was composed of a PCLDMA with a molecular weight of $10000 \mathrm{~g} \mathrm{~mol}^{-1}$ and a $4 \%$ wt of UPy with respect to the weight of PCLDMA. Both components were functionalized with the same methacrylating agent, 2-IEM, to obtain photo-reactive groups. The mixture of PCLDMA and UPyMA was heated up to $45^{\circ} \mathrm{C}$ by a custommade hot plate in a DLP printer. Printed specimens underwent a postcuring of $30 \mathrm{~min}$ in a UV oven (DWS Systems, Italy) in order to ensure the complete crosslinking of the material. An "L" shaped object was printed to simulate the shape of the index finger and the thumb of a robotic hand. The resulting crosslinked polymer was characterized by a homogeneous diffusion of UPy groups into the PCL matrix, provided also by the mixing and printing temperature of $45^{\circ} \mathrm{C}$.

In order to demonstrate self-healing abilities in presence of bulk damage, the section of a printed object was partially cut and was repaired with thermal treatment in an oven for $1 \mathrm{~h}$ at $80^{\circ} \mathrm{C}$, keeping the damaged cross-sections as close as possible. Figure 3 shows the partial cut and the result of this test, ensuring the effectiveness of the formulated system.

DSC analysis was also performed on a printed PCLDMA + UPyMA sample. Compared to the corresponding cast samples, the melting temperature appeared not considerably changed, slightly rising to $55^{\circ} \mathrm{C}$, while the degree of crystallinity increased up to $30 \pm 2 \%$ (Figure 1). However, if the values of standard deviations are considered, this small increase of 5\% in the degree of crystallinity does not seem so significant, comparing cast and printed PCLDMA + UPyMA samples.

To evaluate the mechanical properties of this material, tensile tests were carried out for the cast and printed samples with and without UPyMA. Cast PCLDMA + UPyMA specimens exhibited a maximum tensile strength lower than cast PCLDMA sample without UPyMA (Figure 4a). This can be explained considering that the presence of UPy motifs between PCLDMA chains can reduce their capability to withstand stresses. However, the differences are not so evident and this demonstrates that the presence of UPy moieties did not interfere negatively with mechanical performances. Printed PCLDMA + UPyMA specimens showed a tensile strength similar to cast samples and a small reduction in the values of elongation at break. The recovery of the elongation at break after repairing treatments was not so high, probably due to small defects induced by cutting and self-healing tests, however mechanical properties of repaired PCLDMA+UPyMA printed samples are still suitable for the application in fields such as soft actuators. ${ }^{71,72}$ As regards the elastic modulus, no significant differences were observed between cast samples without and with the incorporation of the UPyMA motifs (Figure 4b). This demonstrates that 


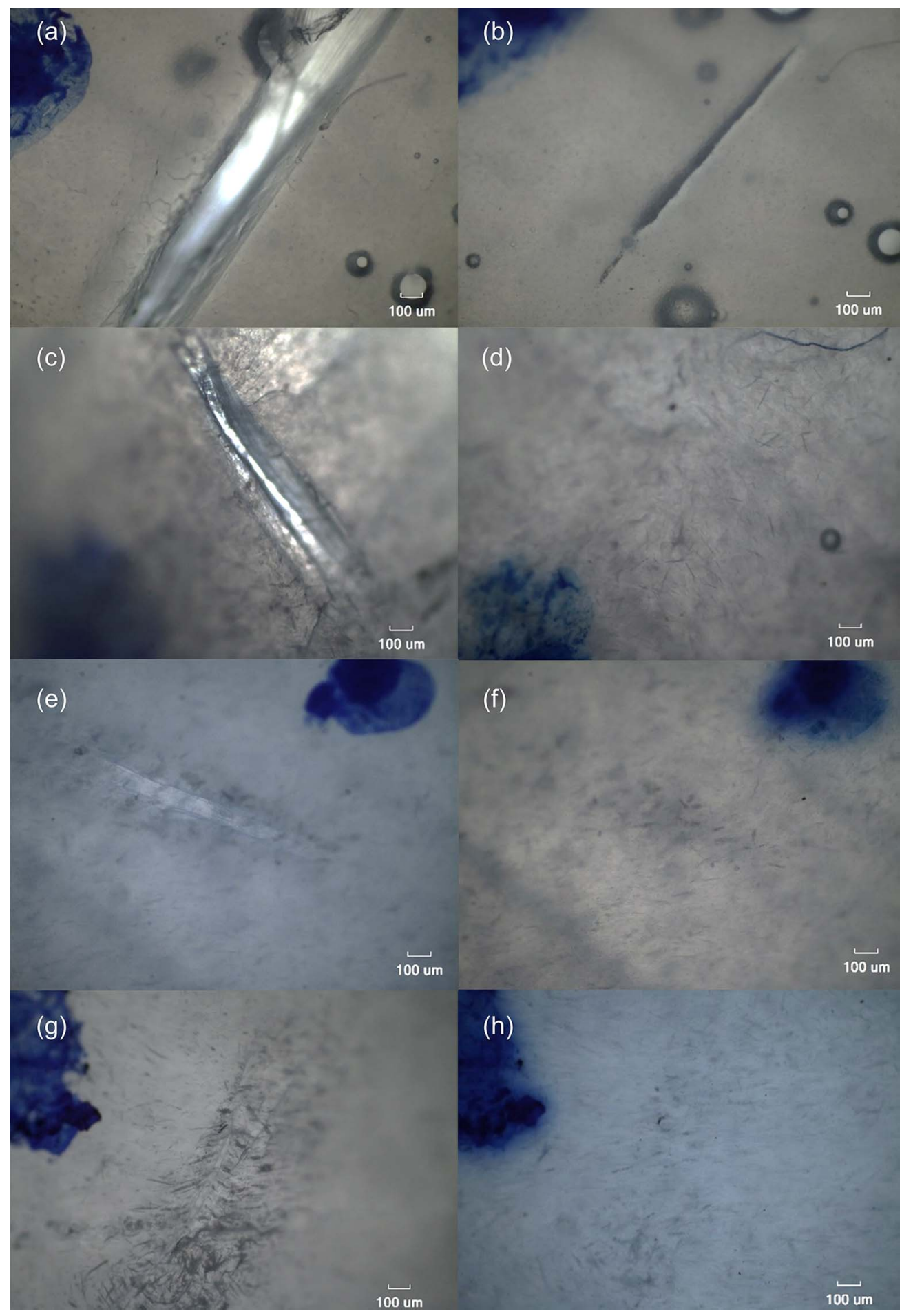

Figure 2. Thermally induced self-healing tests for a sample surface of (a, b) neat PCLDMA, PCLDMA-UPyMA with (c, d) 3\% wt, (e, f) 4\% wt and (g, h) 5\% wt of UPyMA. The tests consisted in producing a scratch with a scalpel blade on the sample surfaces and observing surface scratches by means of microscopy images before $(\mathrm{a}, \mathrm{c}, \mathrm{e}, \mathrm{g})$ and after $(\mathrm{b}, \mathrm{d}, \mathrm{f}, \mathrm{h})$ thermal treatment of $1 \mathrm{~h}$ at $80^{\circ} \mathrm{C}$.

UPyMA units did not affect the level of stiffness of PCLDMA samples, providing the advantage of self-healing abilities. Printed and undamaged PCLDMA+UPyMA samples were also compared to printed and damaged PCLDMA+UPyMA to check the effect of the repairing process on mechanical performances. Considering the elastic modulus, printed and repaired samples showed results comparable to those observed for printed and undamaged samples and even to those obtained from casting (Figure 4b).
Moreover, a healing efficiency $\eta$, which is defined as the ratio between the maximum tensile strength of healed samples and the maximum tensile strength of undamaged samples, ${ }^{73}$ was calculated (Figure 5). The calculated values were similar to those found in the literature for polymeric systems bearing UPyMA motifs and could be even enhanced by increasing the healing time, the temperature of healing ${ }^{74}$ and the waiting time before healing. ${ }^{75}$ This suggests the possibility of further increasing $\eta$ of PCLDMA+UPyMA printed objects 


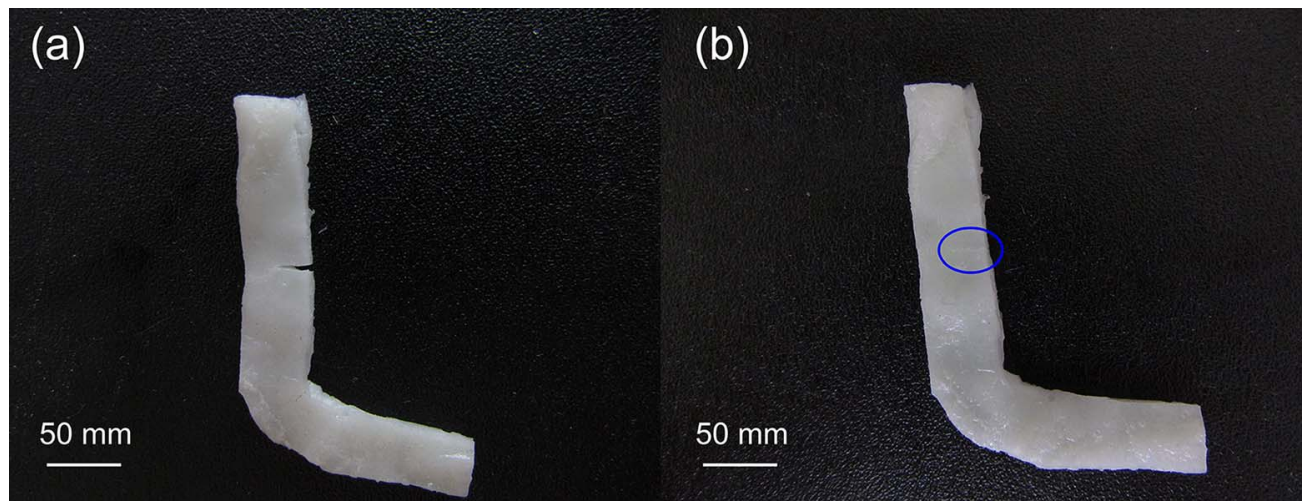

Figure 3. Images of an "L"-shaped object printed using a PCLDMA and UPyMA (4\% wt. with respect to PCLDMA) mixture (a) after a partial cut of its section and (b) after thermal treatment at $80^{\circ} \mathrm{C}$ for $1 \mathrm{~h}$, keeping the cut cross-sections in close contact during thermally self-healing tests, in order to repair the object.

(a)

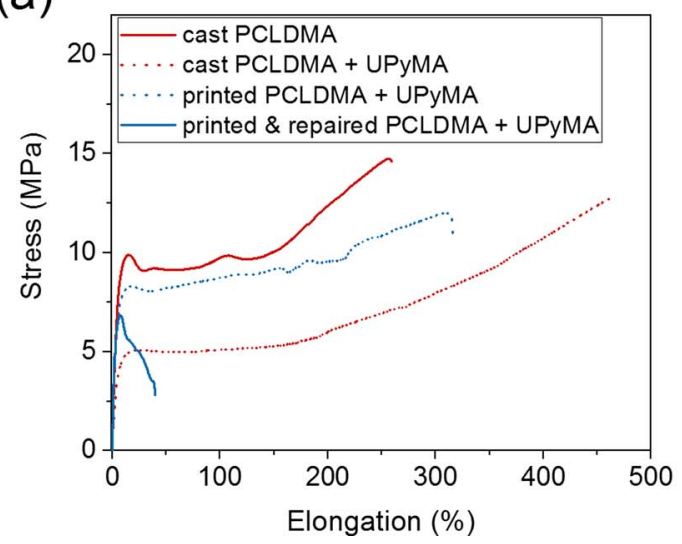

(b)

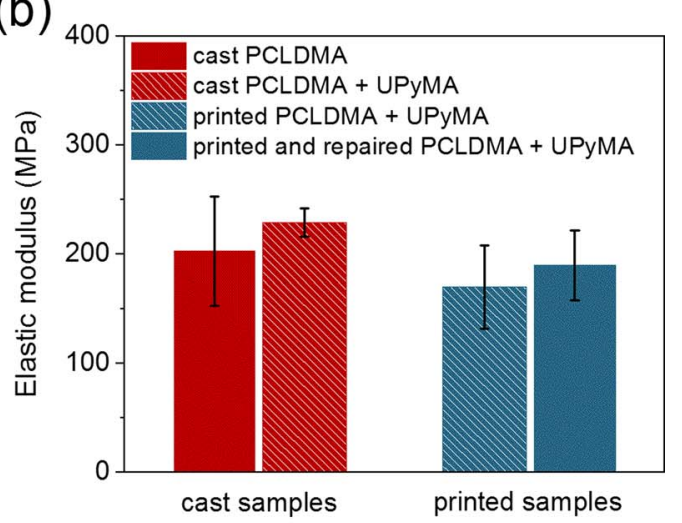

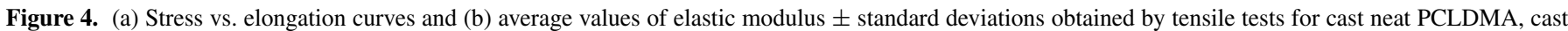
PCLDMA + UPyMA, printed PCLDMA + UPyMA, and printed and repaired PCLDMA + UPyMA.

by performing studies on the waiting time before healing and healing time.

Figure 5 also shows healing efficiency for printed samples higher than those obtained by casting. This can be likely due to a more efficient interpenetration between PCLDMA matrix and UPyMA and more numerous bridges between UPy units at the razor-cut interfaces as shown by a rougher interface at the breaking point for 4D printed samples, when compared to cast ones. ${ }^{76}$

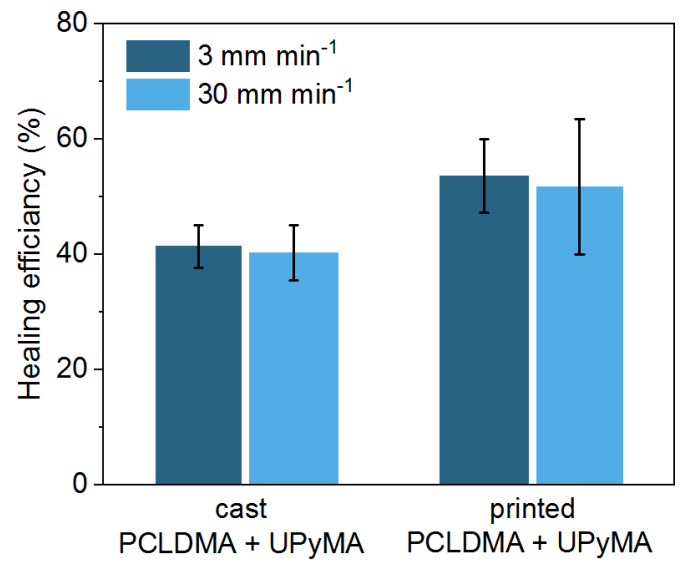

Figure 5. Healing efficiency $\eta$, calculated as the ratio between the maximum tensile strength of healed samples and the maximum tensile strength of undamaged samples measured at two different tensile test speeds ( 3 and $30 \mathrm{~mm}$ $\mathrm{min}^{-1}$ ) for cast and printed PCLDMA + UPyMA samples.
Furthermore, printed objects exhibited good shape memory properties, as shown in Figure 6. Quantitatively, shape memory behavior was evaluated considering two parameters: the recovery ratio $R_{r}$, which is defined as the ability of a polymeric material to memorize the original shape from a temporary deformed shape and the fixity ratio $R_{f}$, which describes the ability to fix a deformation applied during a programming process. For 4D printed PCLDMA-UPyMA samples, a high value of $99.8 \%$ was determined for $R_{f}$ and of $98.6 \%$ for $R_{r}$, respectively demonstrating the efficacy of the programming process and the ability of the material to almost completely recovered its permanent shape.

\section{Conclusions and Future Challenges}

A 4D printable smart material was successfully developed and 4D printed. This material was composed of a thermally activated SMP, capable of self-repairing if damaged. The SME was governed by netpoints consisted of chemical crosslinks, which led to high values of fixity and recovery ratios. UPy units provided an intrinsic self-healing behavior, due to supramolecular hydrogen bonding interactions triggered by heat. A parametric study to select an optimal concentration of UPyMA was carried out. A commercial low-cost DLP printer was modified in order to heat the material during the printing process and to enable a good diffusion of UPy motifs into the PCL matrix. Printed objects showed a remarkable SME and self-repairing abilities in case of surface and bulk damage.

Although the 4D printing technology is quite promising and is expected to find rapidly applications in healthcare fields as well as aerospace and automotive industries, several challenges need to be 


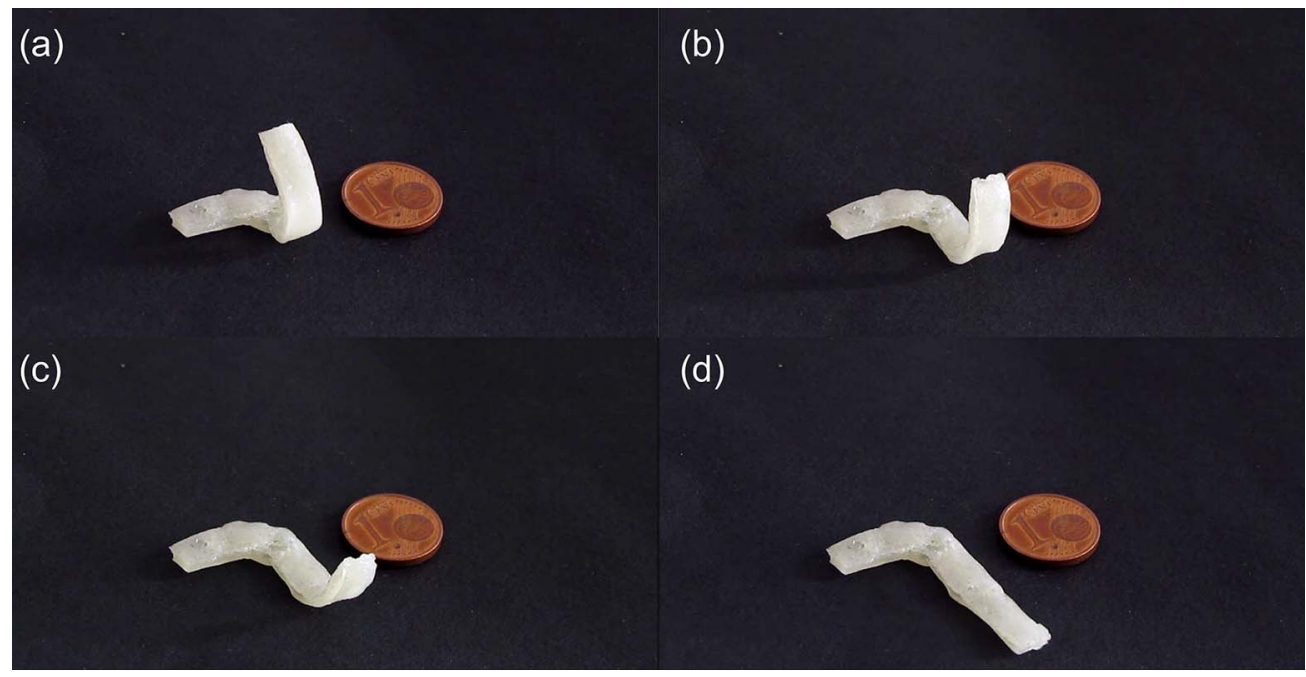

Figure 6. Images showing the shape memory effect for a printed "L"-shaped object composed of PCLDMA and UPyMA (4\% wt with respect to PCLDMA): (a) a temporary shape was firstly obtained and fixed; (b) and (c) the permanent shape was partially regained by means of thermal treatment at $70^{\circ} \mathrm{C}$ and $(\mathrm{d})$ a complete recovery of the permanent shape was obtained after few minutes.

overcome in order to make it widespread. One of the major challenges is the reduction of high initial costs, which can be lowered by increasing the number of companies that develops printers that support 4D printing. From a material-related point of view, other crucial aspects are the limited availability of stimuli responsive materials that can be manufactured by extrusion based or liquid processing 4D printing and the possibility of enlarging the range of mechanical properties showed by these materials.

4D inkjet printing also presents major challenges, the most critical being the limited tridimensionality of printable features. Inkjet manufactured 4D parts are typically characterized by two dimensions largely exceeding the third, as a consequence of the intrinsic limits of this technology. Indeed, the improvement of inkjet tridimensionality is one of the crucial points that must be addressed to really extend the applications range of this technology. Another major problem for inkjet, which is even more challenging than for extrusion based or liquid processing 4D printing, is the selection of materials. In fact, they must be not only stimuli-responsive but also easily jettable. This implies the use of dilute solutions or nanoparticles suspensions, which strongly limit the range of usable materials.

Another important point is also the reversibility of shape changes for the systems mainly diffused so far, which cannot remember the temporary shape. In other words, the commonly used SMPs can be programmed, the temporary shape is fixed and the original shape is regained by applying the stimulus but a new programming cycle has to be applied in order to fix the temporary shape again. Therefore, a great challenge is to prepare SMPs capable of storing the information related to the temporary shape during the recovery of the original shape, thus making unnecessary the continuous re-programming. Moreover, the possibility of fast response times will enable the extensive use of SMPs in the robotic field, where accurate and quick movements are necessary.

\section{Acknowledgments}

The authors would like to thank Mr. Stefano Cagninelli for helping to modify the $3 \mathrm{D}$ printer configuration and changing it for the purpose of this work.

\section{ORCID}

Luca Magagnin (D) https://orcid.org/0000-0001-5553-6441

Marinella Levi (D) https://orcid.org/0000-0001-5921-7202

\section{References}

1. S. Tibbits, Architectural Design, 84(1), 116 (2014).

2. S. Tibbits, TED talk, 2013, https://www.ted.com/talks/skylar_tibbits_the_ emergence_of_4d_printing\#t-20838.

3. Q. Ge, C. K. Dunn, H. J. Qi, and M. L. Dunn, Smart Mater. Struct., 23(9), 094007 (2014).

4. S. Miao, N. Castro, M. Nowicki, L. Xia, H. Cui, X. Zhou, W. Zhu, S.-J. Lee, K. Sarkar, G. Vozzi, Y. Tabata, J. Fisher, and L. G. Zhang, Mater. Today, 20(10), 577 (2017).

5. F. Momeni, S. M. Mehdi Hassani.N, X. Liu, and J. Ni, Mater. Des., 122, 42 (2017)

6. A. Y. Lee, J. An, and C. K. Chua, Engineering, 3(5), 663 (2017).

7. P. Rastogi and B. Kandasubramanian, Chem. Eng. J., 366, 264 (2019)

8. M. Behl, J. Zotzmann, and A. Lendlein, in Shape-Memory Polymers, A. Lendlein, ed., p. 1, Springer Berlin Heidelberg, Berlin, Heidelberg, (2010).

9. L. Sun, W. M. Huang, Z. Ding, Y. Zhao, C. C. Wang, H. Purnawali, and C. Tang, Mater. Des, 33, 577 (2012).

10. G. J. Monkman, Mechatronics, 10(4), 489 (2000).

11. A. Lendlein and S. Kelch, Angew. Chem., Int. Ed., 41(12), 2034 (2002).

12. W. Gao, Y. Zhang, D. Ramanujan, K. Ramani, Y. Chen, C. B. Williams, C. C. L. Wang, Y. C. Shin, S. Zhang, and P. D. Zavattieri, Comput. Aided Des., 69, 65 (2015).

13. W. Wu, W. Ye, Z. Wu, P. Geng, Y. Wang, and J. Zhao, Materials, 10(8), 970 (2017).

14. M. D. Hager, S. Bode, C. Weber, and U. S. Schubert, Prog. Polym. Sci., 49-50, 3 (2015).

15. Y. Yang, Y. Chen, Y. Wei, and Y. Li, Int. J. Adv. Manuf. Tech., 84(9), 2079 (2016).

16. J. A. Lewis, Adv. Funct. Mater., 16(17), 2193 (2006).

17. G. Postiglione, G. Natale, G. Griffini, M. Levi, and S. Turri, Composites, Part A, 76, 110 (2015).

18. S. E. Bakarich, R. Gorkin III, M. I. H. Panhuis, and G. M. Spinks, Macromol. Rapid Commun., 36(12), 1211 (2015).

19. A. Sydney Gladman, E. A. Matsumoto, R. G. Nuzzo, L. Mahadevan, and J. A. Lewis, Nat. Mater, 15, 413 (2016).

20. H. Wei, Q. Zhang, Y. Yao, L. Liu, Y. Liu, and J. Leng, ACS Appl. Mater. Interfaces, 9(1), 876 (2017)

21. S. D. Hoath, Editor, Fundamentals of Inkjet Printing: The Science of Inkjet and Droplets, Wiley-VCH Verlag GmbH \& Co., Weinheim, Germany (2016).

22. O. Brand, G. K. Fedder, C. Hierold, J. G. Korvink, and O. Tabata, Editors, Inkjetbased Micromanufacturing, Wiley-VCH Verlag GmbH \& Co., Weinheim, Germany (2012).

23. E. Tekin, P. J. Smith, and U. S. Schubert, Soft Matter, 4(4), 703 (2008),

24. B.-J. de Gans, P. C. Duineveld, and U. S. Schubert, Adv. Mater., 16(3), 203 (2004).

25. A. Atkinson, J. Doorbar, A. Hudd, D. L. Segal, and P. J. White, J. Sol-Gel Sci. Technol., 8(1), 1093 (1997).

26. A. Matavž, R. C. Frunză, A. Drnovšek, V. Bobnar, and B. Malič, J. Mater. Chem. C, 4(24), 5634 (2016)

27. B. Bao, M. Li, Y. Li, J. Jiang, Z. Gu, X. Zhang, L. Jiang, and Y. Song, Small, 11(14), 1649 (2015).

28. N. C. Raut and K. Al-Shamery, J. Mater. Chem. C, 6(7), 1618 (2018)

29. X. Liu, T.-J. Tarn, F. Huang, and J. Fan, Particuology, 19, 1 (2015).

30. S. Sharma, S. S. Pande, and P. Swaminathan, RSC Adv., 7(63), 39411 (2017).

31. B. Derby, J. Eur. Ceram. Soc., 31(14), 2543 (2011). 
32. A. Capasso, A. E. Del Rio Castillo, H. Sun, A. Ansaldo, V. Pellegrini, and F. Bonaccorso, Solid State Commun., 224, 53 (2015).

33. O.-S. Kwon, H. Kim, H. Ko, J. Lee, B. Lee, C.-H. Jung, J.-H. Choi, and K. Shin, Carbon, 58, 116 (2013).

34. T. Xu, J. Jin, C. Gregory, J. J. Hickman, and T. Boland, Biomaterials, 26(1), 93 (2005).

35. Z. Yin, Y. Huang, N. Bu, X. Wang, and Y. Xiong, Chin. Sci. Bull., 55(30), 3383 (2010).

36. C.-T. Wang, K.-Y. Huang, D. T. W. Lin, W.-C. Liao, H.-W. Lin, and Y.-C. Hu, Sensors, 10(5), 5054 (2010)

37. M. Rieu, M. Camara, G. Tournier, J.-P. Viricelle, C. Pijolat, N. F. de Rooij, and D. Briand, Sens. Actuators, B, 236, 1091 (2016).

38. K. Yamada, T. G. Henares, K. Suzuki, and D. Citterio, Angew. Chem., Int. Ed., 54(18), $5294(2015)$

39. H. Gorter, M. J. J. Coenen, M. W. L. Slaats, M. Ren, W. Lu, C. J. Kuijpers, and W. A. Groen, Thin Solid Films, 532, 11 (2013).

40. J. Vaithilingam, E. Saleh, L. Körner, R. D. Wildman, R. J. M. Hague, R. K. Leach and C. J. Tuck, Mater. Des., 139, 81 (2018).

41. S. H. Ko, J. Chung, N. Hotz, K. H. Nam, and C. P. Grigoropoulos, J. Micromech Microeng., 20(12), 125010 (2010).

42. C. Xu, W. Chai, Y. Huang, and R. R. Markwald, Biotechnol. Bioeng., 109(12), 3152 (2012).

43. B. Derby, Engineering, 1(1), 113 (2015).

44. R. Singh, J Mech Sci Technol, 25(4), 1011 (2011).

45. Z. Ding, C. Yuan, X. Peng, T. Wang, H. J. Qi, and M. L. Dunn, Sci. Adv., 3(4), e1602890 (2017).

46. D. Raviv, W. Zhao, C. McKnelly, A. Papadopoulou, A. Kadambi, B. Shi, S. Hirsch, D. Dikovsky, M. Zyracki, C. Olguin, R. Raskar, and S. Tibbits, Sci. Rep., 4, 7422 (2014).

47. J. Wu, C. Yuan, Z. Ding, M. Isakov, Y. Mao, T. Wang, M. L. Dunn, and H. J. Qi, Sci. Rep., 6, 24224 (2016)

48. Y. L. Yap and W. Y. Yeong, Virtual Phys. Prototyp., 10(2), 91 (2015).

49. W. Marius, C. Tian, and S. Kristina, 3D Print. Addit. Manuf., 4(3), 133 (2017).

50. N. A. Meisel, A. M. Elliott, and C. B. Williams, J. Intell. Mater. Syst. Struct., 26(12), 1498 (2015).

51. C. L. van Oosten, C. W. M. Bastiaansen, and D. J. Broer, Nat. Mater, 8, 677 (2009)

52. J. Bito, R Bahr, J. Hester, J. Kimionis, A Nauroze, W. Su, B. Tehrani, and M. M. Tentzeris, Inkjet-/3D-/4D-printed autonomous wearable RF modules for biomonitoring, positioning and sensing applications, Proc. SPIE 10194, Micro- and Nanotechnology Sensors, Systems, and Applications IX, 101940Z (18 May 2017).
53. J. Kimionis, M. Isakov, B. S. Koh, A. Georgiadis, and M. M. Tentzeris, IEEE Trans. Microwave Theory Tech., 63(12), 4521 (2015).

54. S. A. Nauroze, L. Novelino, M. M. Tentzeris, and G. H. Paulino, 2017 IEEE MTT-S International Microwave Symposium (IMS), Honololu, HI, p. 1575, (2017).

55. A. Adeyeye, A. Eid, J. Hester, T.-H. Lin, S. A. Nauroze, B. Tehrani, and M. M. Tentzeris, Int. J. High Speed Electron. Syst., 27(01n02), 1840012 (2018).

56. X. Peng, T. Liu, Q. Zhang, C. Shang, Q.-W. Bai, and H. Wang, Adv. Funct. Mater. 27(33), 1701962 (2017).

57. M. Zarek, M. Layani, I. Cooperstein, E. Sachyani, D. Cohn, and S. Magdassi, Adv. Mater, 28(22), 4449 (2016).

58. Y.-C. Li, Y. S. Zhang, A. Akpek, S. R. Shin, and A. Khademhosseini, Biofabrication, 9(1), 012001 (2016)

59. G. Villar, A. D. Graham, and H. Bayley, Science, 340(6128), 48 (2013).

60. M. Zarek, N. Mansour, S. Shapira, and D. Cohn, Macromol. Rapid Commun., 38(2), 1600628 (2017)

61. M. Zarek, M. Layani, S. Eliazar, N. Mansour, I. Cooperstein, E. Shukrun, A. Szlar, D. Cohn, and S. Magdassi, Virtual Phys. Prototyp., 11(4), 263 (2016)

62. Y. Y. C. Choong, S. Maleksaeedi, H. Eng, J. Wei, and P.-C. Su, Mater. Des., 126, 219 (2017).

63. C. Laschi, B. Mazzolai, V. Mattoli, M. Cianchetti, and P. Dario, Bioinspiration Biomimetics, 4(1), 015006 (2009).

64. D. Rus and M. T. Tolley, Nature, 521, 467 (2015)

65. S. Kim, C. Laschi, and B. Trimmer, Trends Biotechnol., 31(5), 287 (2013).

66. R. Suriano, L. Brambilla, M. Tommasini, and S. Turri, Polym. Adv. Technol., 29(12), 2899 (2018)

67. M. Messori, M. Degli Esposti, K. Paderni, S. Pandini, S. Passera, T. Riccò, and M. Toselli, J. Mater. Sci., 48(1), 424 (2013).

68. K. Yamauchi, J. R. Lizotte, and T. E. Long, Macromolecules, 36(4), 1083 (2003).

69. A. Lendlein, A. M. Schmidt, M. Schroeter, and R. Langer, J. Polym. Sci., Part A: Polym. Chem. 43(7), 1369 (2005).

70. M. Behl and A. Lendlein, Mater. Today, 10(4), 20 (2007).

71. R. V. Martinez, A. C. Glavan, C. Keplinger, A. I. Oyetibo, and G. M. Whitesides, Adv. Funct. Mater. 24(20), 3003 (2014).

72. B. C. Tee, C. Wang, R. Allen, and Z. Bao, Nat. Nanotechnol., 7(12), 825 (2012).

73. M. Wei, M. Zhan, D. Yu, H. Xie, M. He, K. Yang, and Y. Wang, ACS Appl. Mater. Interfaces, 7(4), 2585 (2015).

74. Y. Wu, L. Wang, X. Zhao, S. Hou, B. Guo, and P. X. Ma, Biomaterials, 104, 18 (2016)

75. G. M. L. van Gemert, J. W. Peeters, S. H. M. Söntjens, H. M. Janssen, and A. W. Bosman, Macromol. Chem. Phys., 213(2), 234 (2012).

76. M. Invernizzi, S. Turri, M. Levi, and R. Suriano, Eur. Polym. J., 101, 169 (2018). 